\title{
Resveratrol suppresses vascular endothelial growth factor secretion via inhibition of CXC-chemokine receptor 4 expression in ARPE-19 cells
}

\author{
HYEMIN SEONG ${ }^{1}$, JINHYUN RYU ${ }^{1}$, JOO YEON JEONG ${ }^{1}$, IN YOUNG CHUNG ${ }^{2}$, YONG-SEOP HAN ${ }^{2}$, \\ SOO HYUN HWANG ${ }^{3}$, JONG MOON PARK ${ }^{2}$, SANG SOO KANG $^{1}$ and SEONG WOOK SEO ${ }^{2}$
}

\begin{abstract}
Departments of ${ }^{1}$ Anatomy and Convergence Medical Science, ${ }^{2}$ Ophthalmology and ${ }^{3}$ Neurosurgery, Institute of Health Sciences, School of Medicine, Gyeongsang National University, Jinju, Gyeongnam 660-751, Republic of Korea
\end{abstract}

Received June 24, 2014; Accepted March 5, 2015

DOI: $10.3892 / \mathrm{mmr} .2015 .3518$

\begin{abstract}
The present study characterizes the effects of resveratrol (Res) on vascular endothelial growth factor (VEGF) secretion in retinal pigment epithelial (RPE) cells. ARPE-19 cells were treated with $\mathrm{CoCl}_{2}$, a hypoxia mimetic agent. $\mathrm{CoCl}_{2}$ treatment increased protein levels of hypoxia inducible factor- $1 \alpha$ (HIF-1 $\alpha)$ and CXC-chemokine receptor 4 (CXCR4), and secretion of VEGF. To confirm the effects of Res on VEGF secretion, the human umbilical vein endothelial cell tube formation assay was performed with conditioned medium from Res-treated ARPE-19 cells. The well-known antioxidant Res effectively blocked these effects and reduced phosphorylation of nuclear factor (NF)- $\kappa \mathrm{B}$, an upstream activator of CXCR4. Furthermore, Res also suppressed VEGF secretion induced by SDF-1, a ligand of CXCR4. Conditioned medium from Res-treated ARPE-19 cells clearly suppressed tube formation compared with hypoxia-treated conditioned medium. The results demonstrated that Res inhibited the hypoxia mimetic $\mathrm{CoCl}_{2}$-induced expression of VEGF in ARPE-19 cells. Res suppressed CXCR4 expression through decreased phosphorylation of $\mathrm{NF}-\kappa \mathrm{B}$, resulting in downregulation of VEGF secretion.
\end{abstract}

Correspondence to: Professor Seong Wook Seo, Department of Ophthalmology, Institute of Health Sciences, School of Medicine, Gyeongsang National University, 15 Jinju-daero 816 Beon-gil, Jinju, Gyeongnam 660-751, Republic of Korea

E-mail: stramast@naver.com

Professor Sang Soo Kang, Department of Anatomy and Convergence Medical Science, Institute of Health Sciences, School of Medicine, Gyeongsang National University, 15 Jinju-daero 816 Beon-gil, Jinju, Gyeongnam 660-751, Republic of Korea

E-mail:kangss@gnu.ac.kr

Key words: neovascularization, vascular endothelial growth factor, CXC-chemokine receptor 4, resveratrol, ARPE-19, hypoxia

\section{Introduction}

Neovascularization is a major cause of common eye diseases, such as age-related macular degeneration (AMD), diabetic retinopathy, and retinopathy of prematurity (ROP) that result in blindness (1). New vessels that are generated by neovascularization usually undergo repeated regeneration, which can easily lead to bleeding and vessel destruction due to stress caused by increased permeability. A number of the pathways that result in neovascularization have been identified (2). The majority of these pathways, such as the protein kinase B (AKT) pathway, the nuclear factor of activated T-cells (NFAT) signaling pathway, and the Nox2-generated reactive oxygen species (ROS) pathway, are associated with increased vascular endothelial growth factor (VEGF) expression (3-5). Therefore, VEGF is a major component of the neovascularization process.

VEGF-A, also termed VEGF, is a member of the cysteine-knot superfamily of growth factors. The characteristics of VEGF are determined by a cysteine residue $(6,7)$. Increased secretion of VEGF induces neovascularization, and anti-VEGF therapy with bevacizumab is effective in the suppression of neovascularization (8). Certain conditions, such as diabetes, trauma and infection (9), can induce hypoxia, which results in the increased expression of VEGF in the eye (10). Secretion of VEGF is also generally observed in cancer cells. In certain types of cancer, the cell secretes VEGF locally to stimulate the surrounding endothelial cells for neovascularization (11). Retinal pigment epithelial (RPE) cells are a source of VEGF in the eye (12). RPE cells form a monolayer structure at the back of the eye, and supply nutrients and remove waste from the surrounding tissues. Although there are several pathways that regulate VEGF expression, the present study focuses on the interaction between $\mathrm{CXC}$-chemokine receptor 4 (CXCR4) and VEGF.

CXCR4 is a well-known hypoxia-related protein (13). Studies of cancer cells revealed that CXCR4 expression is increased during hypoxia and mediates VEGF upregulation $(14,15)$. This suggested that CXCR4 could be a useful target for inhibition of neovascularization.

Resveratrol (Res) is a phytoalexin present in grapes, red wine and other food products. Res has significant effects 
on inflammation, apoptosis and neovascularization $(16,17)$. Furthermore, Res is particularly effective as an antioxidant in hypoxic conditions (18). Res also prevents or mitigates the effects of eye diseases, such as retinal detachment and diabetic retinopathy (19-22). The main target of Res is nuclear factor $(\mathrm{NF})-\kappa \mathrm{B}$, which is a major factor of the SDF-1/CXCR4 axis (23).

In this study, the major regulators of hypoxia-induced VEGF secretion in the ARPE-19 human retinal epithelial cell line were characterized. The present study focused on the $\mathrm{NF}-\kappa \mathrm{B}, \mathrm{CXCR} 4$ and VEGF pathways, and the effect of Res on neovascularization induced by hypoxia.

\section{Materials and methods}

Reagents. High-glucose Dulbecco's modified Eagle's medium F12 (DMEM-F12), penicillin, streptomycin and fetal bovine serum (FBS) were purchased from Gibco (Grand Island, NY, USA). TRIzol reagent was purchased from Invitrogen Life Technologies (Carlsbad, CA, USA). Res was purchased from Tocris (Ellisville, MO, USA), SDF-1 was purchased from R\&D systems (Minneapolis, MN, USA) and dimethylsulfoxide (DMSO) was purchased from Amresco (Solon, OH, USA). Antibodies specific for hypoxia inducible factor-1 $\alpha$ (HIF-1 $\alpha$; cat. no. 3716S) and phospho-NF- $\mathrm{B}$ (cat. no. 3033S) were purchased from Cell Signaling Technology Inc. (Danvers, MA, USA), anti-CXCR4 antibodies (cat. no. ab2074) were purchased from Abcam (Cambridge, UK), and anti- $\alpha$-tubulin antibodies (cat. no. T5168) were purchased from Sigma-Aldrich (St. Louis, MO, USA). Ammonium pyrrolidinethiocarbamate (PDTC), NF- $\kappa$ B inhibitor and AMD3100 (a CXCR4 antagonist), were purchased from Sigma-Aldrich. BD Matrigel ${ }^{\mathrm{TM}}$ Basement Membrane Matrix was purchased from BD Biociences (Bedford, MA, USA). Resveratrol was dissolved in DMSO, PDTC and AMD3100 were dissolved in water, and SDF-1 was dissolved in phosphate-buffered saline (PBS).

Cell culture and treatment. ARPE-19 cells (American Type Culture Collection, Manassas, VA, USA) were cultured in DMEM F12 supplemented with 10\% FBS, $100 \mathrm{U} / \mathrm{ml}$ penicillin, and $100 \mu \mathrm{g} / \mathrm{ml}$ streptomycin at $37^{\circ} \mathrm{C}$ in an atmosphere with $5 \% \mathrm{CO}_{2}$ at $70 \%$ confluence. For some experiments, ARPE-19 cells were cultured in DMEM-F12 with $0.1 \%$ FBS in the presence of designated concentrations of VEGF protein and/or Res (10-50 $\mu \mathrm{M})$. Cells were exposed to $\mathrm{CoCl}_{2}$ for $6 \mathrm{~h}$ prior to western blotting. Cells were grown as monolayer.

Western blotting. ARPE-19 cells were plated on 100-mm dishes at $1 \times 10^{6}$ cells/dish. Following treatment, cells were harvested, centrifuged at $890 \mathrm{x} \mathrm{g}$ for $1 \mathrm{~min}$, and lysed in lysis buffer [50 mM Tris- $\mathrm{Cl}$ ( $\mathrm{pH} 8.0$ ), $150 \mathrm{mM} \mathrm{NaCl}, 0.1 \%$ sodium dodecyl sulfate (SDS) and $0.02 \%$ sodium azide, which were purchased from Amresco; $1 \%$ NP-40 and 0.5\% sodium deoxycholate, which were purchased from Sigma-Aldrich, and proteinase inhibitor cocktail (containing phenylmethylsulphonyl fluoride, $100 \mu \mathrm{g} / \mathrm{ml}$; aprotinin, $1 \mu \mathrm{g} / \mathrm{ml}$; leupeptin, $0.5 \mu \mathrm{g} / \mathrm{ml}$; Roche, Nutley, NJ, USA)]. Total protein concentration was determined using a bicinchoninic acid protein assay system (Thermo Fisher Scientific, Waltham, MA, USA). An equal volume of $4 \mathrm{X}$ SDS sample buffer was added to $30 \mu \mathrm{g}$ of protein extract and the samples were boiled for $5 \mathrm{~min}$. Equivalent quantities of total protein (15-20 $\mu \mathrm{g}$ ) were separated by SDS-PAGE on $8-10 \%$ polyacrylamide gels and then transferred to nitrocellulose membranes (GE Healthcare, Little Chalfont, UK) at $15 \mathrm{~V}$ for $30 \mathrm{~min}$ using a semi-dry transfer apparatus (Bio-Rad Laboratories, Hercules, CA, USA) submerged in transfer buffer (25 mM Tris, $192 \mathrm{mM}$ glycine, 20\% methanol; $\mathrm{pH}$ 8.3). The membrane was blocked with $5 \%$ skimmed milk in $0.1 \%$ Tween-20/Tris-buffered saline (TBST) and then incubated with anti-CXCR4 $(1: 1,000)$, anti-HIF-1 $\alpha(1: 1,000)$, anti-phospho- NF- $\mathrm{B}(1: 1,000)$ or anti- $\alpha$-tubulin $(1: 10,000)$ antibody at $4^{\circ} \mathrm{C}$ overnight. Subsequently, the blot was washed in TBST and incubated with goat anti-rabbit (cat. no. 31430) and goat anti-mouse (cat. no. 31460) immunoglobulin G secondary antibodies (Thermo Fisher Scientific; 1:10,000 dilution) for $45 \mathrm{~min}$ at room temperature. After washing, immunoreactivity was detected by chemiluminescence (ECL; Advansta, Menlo Park, CA, USA) using the LAS 3000 instrument (Fujifilm, Tokyo, Japan). The band intensities were assessed by Multi Gauge 3.0 software (Fujifilm).

Enzyme-linked immunosorbent assay (ELISA). ARPE-19 cells were plated on 6 -well cell culture plates at $5 \times 10^{4}$ cells/well. Cell culture media were supplemented with $1 \%$ FBS, $100 \mathrm{U} / \mathrm{ml}$ penicillin and $100 \mu \mathrm{g} / \mathrm{ml}$ streptomycin. VEGF secretion levels were measured using a commercial human VEGF-ELISA kit from Invitrogen Life Technologies (Camarillo, CA, USA), according to the manufacturer's instructions.

Human umbilical vein endothelial cell (HUVEC) tube formation assay. ARPE-19 cells were cultured in DMEM-F12 media with $0.1 \% \mathrm{FBS}, 100 \mathrm{U} / \mathrm{ml}$ penicillin and $100 \mu \mathrm{g} / \mathrm{ml}$ streptomycin at $37^{\circ} \mathrm{C}$ and $5 \% \mathrm{CO}_{2}$ in the presence of the designated concentrations of Res $(50 \mu \mathrm{M})$. ARPE-19 cells were classified into three groups: Control [Normoxia conditioned medium (CM)], $1 \% \mathrm{O}_{2}$ hypoxia-treatment $($ Hypoxia $\mathrm{CM})$ and hypoxia $+\operatorname{Res}(50 \mu \mathrm{M})$ co-treatment (Hypoxia + Res CM). Cells were exposed to $1 \%$ $\mathrm{O}_{2}$ for $24 \mathrm{~h}$ in a hypoxia chamber (MCO-5M; Sanyo, Osaka, Japan). The CM obtained from ARPE-19 cells was transferred to HUVECs (PromoCell, Heidelberg, Germany) that were seeded on 24-well plates coated with Matrigel $^{\mathrm{TM}}$ at $1 \times 10^{5}$ cells/well. CM-treated HUVECs were incubated for $48 \mathrm{~h}$. Tube formation was analyzed by light microscopy (U-LH100HG; Olympus Corporation, Tokyo, Japan) and ImageJ $1.46 \mathrm{r}$ software (National Institutes of Health, Bethesda, MD, USA).

Statistical analysis. Data are expressed as the mean \pm standard error of the mean. One-way analysis of variance was performed using Dunnett's post-test. (Prism 5; GraphPad Software, La Jolla, CA, USA). P $<0.05$ was considered to indicate a statistically significant difference.

\section{Results}

HIF-1 $\alpha$ and CXCR4 protein expression, and VEGF secretion profiles ARPE-19 cells treated with a hypoxia mimetic agent. To confirm hypoxia-induced changes in protein expression in ARPE-19 cells, cells were treated with $\mathrm{CoCl}_{2}(100 \mu \mathrm{M})$ a hypoxia mimetic agent for $24 \mathrm{~h}$. HIF- $1 \alpha$ and CXCR4 protein levels were determined by western blotting. Expression of the 
hypoxia marker HIF-1 $\alpha$ was increased by $\sim 3.5$-fold at $3 \mathrm{~h}$ and peaked by $\sim 5$-fold at $12 \mathrm{~h}$. CXCR 4 protein levels also increased at $6 \mathrm{~h}$ (Fig. 1A). VEGF secretion, determined by ELISA, was significantly increased at $12 \mathrm{~h}$ and further increased by $>5$-fold at $24 \mathrm{~h}$ (Fig. 1B). These results showed that CXCR4 protein was expressed in ARPE-19 cells, and that expression of HIF-1 $\alpha$, CXCR4 and VEGF proteins increased after hypoxia mimetic treatment, which were similar to the results found under $1 \% \mathrm{O}_{2}$ hypoxic conditions.

Res suppressed HIF-1a protein expression, $N F-\kappa B$ phosphorylation, CXCR4 protein expression, and VEGF secretion in ARPE-19 cells treated with a hypoxia mimetic agent. To evaluate the effects of the antioxidant Res on the increased expression of CXCR4 protein induced by $\mathrm{CoCl}_{2}$, cells were pre-treated with Res at three different concentrations $(10-50 \mu \mathrm{M})$. After $2 \mathrm{~h}, \mathrm{CoCl}_{2}(100 \mu \mathrm{M})$ was added for a further $6 \mathrm{~h}$. The expression of phosphorylated NF- $\kappa \mathrm{B}$ protein, a major target of Res, was also examined. Res treatment reversed the $\mathrm{CoCl}_{2}$-induced increases in the levels of HIF-1 $\alpha, \mathrm{p}-\mathrm{NF}-\kappa \mathrm{B}$ and CXCR4 protein expression in a dose-dependent manner. The levels of HIF- $1 \alpha, \mathrm{p}-\mathrm{NF}-\kappa \mathrm{B}$ and CXCR4 protein expression were reduced by $\sim 80,60$ and $50 \%$ by $50 \mu \mathrm{M}$ resveratrol, respectively (Fig. 2A), and similar effects on VEGF secretion were observed (Fig. 2B). These results showed that $50 \mu \mathrm{M}$ Res effectively inhibited the phosphorylation of $N F-\kappa B$, expression of HIF- $1 \alpha$ and CXCR4, and secretion of VEGF.

PDTC and AMD3100 suppress VEGF secretion in hypoxia mimetic-treated ARPE-19 cells. To investigate the effects of $\mathrm{NF}-\kappa \mathrm{B}$ and CXCR4 on VEGF secretion, cells were treated with PDTC (10 and $20 \mu \mathrm{M})$ and AMD3100 (100 and $300 \mu \mathrm{M})$ for $12 \mathrm{~h}$ in combination with $\mathrm{CoCl}_{2}$. PDTC and AMD3100 treatments dose-dependently reduced the $\mathrm{CoCl}_{2}$-induced VEGF secretion respectively (Fig. 3). These results showed that Res is able to suppress VEGF secretion through inhibition of $\mathrm{NF}-\kappa \mathrm{B}$ and $\mathrm{CXCR} 4$

Res suppresses SDF-1-induced VEGF secretion. As inhibition of CXCR4 suppressed VEGF secretion, it was then analyzed whether Res can suppress VEGF secretion induced by ligand-receptor interaction of CXCR4. Cells were treated with SDF-1 $(30 \mathrm{ng} / \mathrm{ml})$, a ligand of CXCR4, alone or in combination with Res $(50 \mu \mathrm{M})$ for $12 \mathrm{~h}$. SDF-1 induced-secretion of VEGF in ARPE-19 cells was suppressed by co-treatment with Res (50 $\mu \mathrm{M})$ (Fig. 4).

Suppression of HUVEC tube formation by CM from Res-treated ARPE-19 cells. To indirectly investigate the effect of Res on neovascularization the HUVEC tube formation assay was used. ARPE-19 cells were classified into three groups: Control (Normoxia CM), $1 \% \mathrm{O}_{2}$ hypoxia-treatment (Hypoxia CM); and hypoxia+Res $(50 \mu \mathrm{M})$ co-treatment (Hypoxia+Res CM). After $24 \mathrm{~h}$ incubation, CM from the three experimental groups was transferred to HUVECs and the cells were incubated for $48 \mathrm{~h}$. HUVECs treated with Hypoxia CM showed an increase in the number of branch points compared with cells treated with Normoxia CM. HUVECs treated with Hypoxia+Res CM showed a significant decrease in the number of branch points HUVECs treated
A

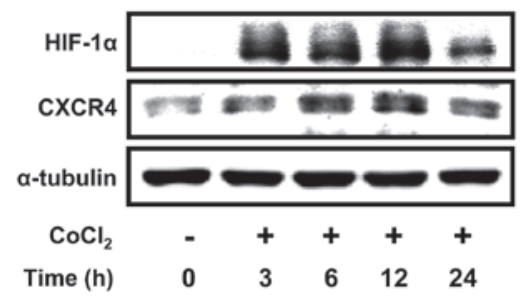

B

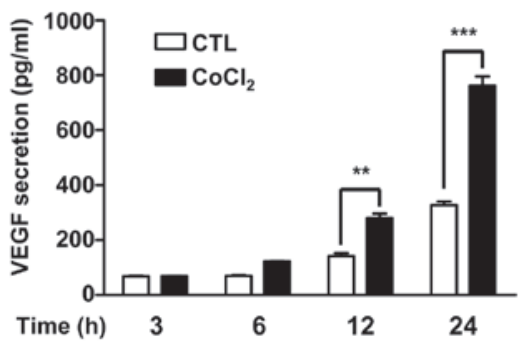

Figure 1. HIF-1 $\alpha$ and CXCR4 protein expression, and VEGF secretion in ARPE-19 cells treated with a hypoxia mimetic agent. Cells were treated with $\mathrm{CoCl}_{2}(100 \mu \mathrm{M})$ for $24 \mathrm{~h}$. (A) Levels of HIF-1 $\alpha$ and CXCR4 protein expression in ARPE-19 cells were measured by western blotting. (B) Levels of VEGF secretion in ARPE-19 cells were determined by enzyme-linked immunosorbent assay. Data are represented as the mean \pm standard error of the mean and analyzed using one-way analysis of variance with Dunnett's post-test $(\mathrm{n}=3) .{ }^{* *} \mathrm{P}<0.01$ and ${ }^{* * *} \mathrm{P}<0.001$. HIF-1 $\alpha$, hypoxia inducible factor- $1 \alpha$; CXCR4, CXC-chemokine receptor 4; VEGF, vascular endothelial growth factor.

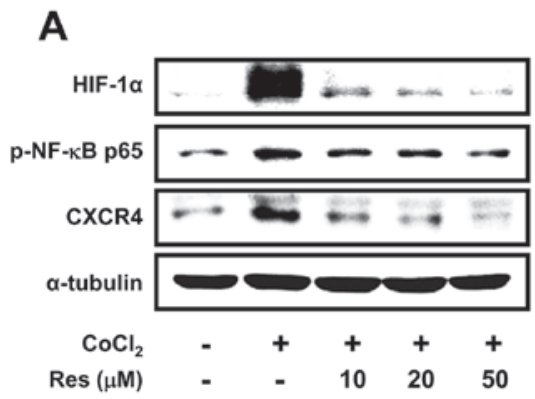

B

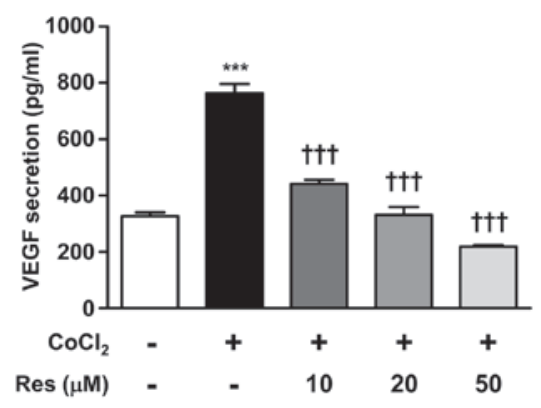

Figure 2. Effects of Res on NF- $\kappa \mathrm{B}$ phosphorylation, and CXCR4 and VEGF protein expression in ARPE-19 cells treated with a hypoxia mimetic agent. (A) Cells were pre-treated with Res $(10-50 \mu \mathrm{M})$ for $2 \mathrm{~h}$ and then co-treated with $\mathrm{CoCl}_{2}$ $(100 \mu \mathrm{M})$ and Res $(10-50 \mu \mathrm{M})$ for $6 \mathrm{~h}$. Levels of p-NF- $\kappa \mathrm{B}$ p65 NF- $\kappa \mathrm{B}$ p65, and CXCR4 protein expression in ARPE-19 cells were determined by western blotting. (B) Cells were pre-treated with Res $(10-50 \mu \mathrm{M})$ for $2 \mathrm{~h}$ and then co-treated with $\mathrm{CoCl}_{2}(100 \mu \mathrm{M})$ and Res $(10-50 \mu \mathrm{M})$ for $24 \mathrm{~h}$. VEGF secretion in ARPE-19 cells was measured by enzyme-linked immunosorbent assay. Data are represented as the mean \pm standard error of the mean and analyzed using one-way ANOVA with Dunnett's post-test $(n=3) .{ }^{* * *} \mathrm{P}<0.001$ vs. control and ${ }^{\dagger \dagger} \mathrm{P}<0.001$ vs. $\mathrm{CoCl}_{2}$. NF- $\kappa \mathrm{B}$, nuclear factor- $\kappa \mathrm{B} ; \mathrm{CXCR} 4, \mathrm{CXC}$-chemokine receptor 4; VEGF, vascular endothelial growth factor; Res, resveratrol. 


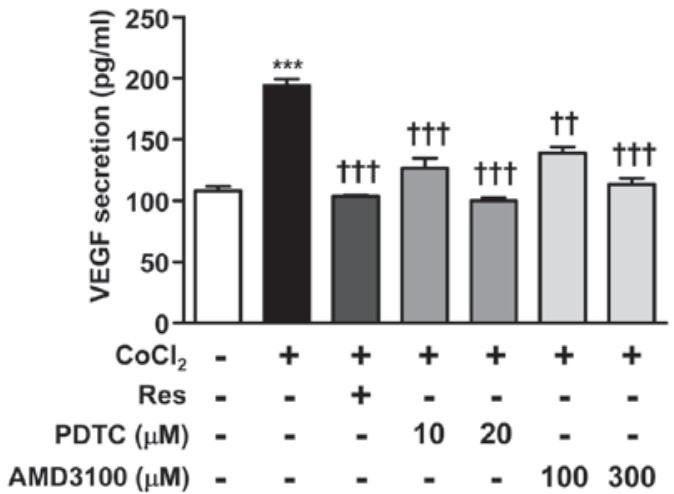

Figure 3. Inhibition of NF- $\kappa \mathrm{B}$ and CXCR4 affect VEGF secretion in hypoxia mimetic treated ARPE-19 cells. Cells were co-treated $\mathrm{CoCl}_{2}(100 \mu \mathrm{M})$ with PDTC $(10-20 \mu \mathrm{M})$, AMD3100 $(100-300 \mu \mathrm{M})$ or Res $(50 \mu \mathrm{M})$ for $12 \mathrm{~h}$. VEGF secretion in ARPE-19 cells was measured by enzyme-linked immunosorbent assay. Data are represented as mean \pm standard error of the mean and analyzed using one-way analysis of variance with Dunnett's post-test $(n=3)$. ${ }^{* * * *} \mathrm{P}<0.001$ vs. control, ${ }^{\dagger} \mathrm{P}<0.01$ vs. $\mathrm{CoCl}_{2}$ and ${ }^{\dagger \dagger} \mathrm{P}<0.001$ vs. $\mathrm{CoCl}_{2}$. NF- ${ }^{\circ B}$, nuclear factor- $\mathrm{\kappa}$; CXCR4, CXC-chemokine receptor 4; VEGF, vascular endothelial growth factor; PTDC, Ammonium pyrrolidinethiocarbamate; Res, resveratrol.

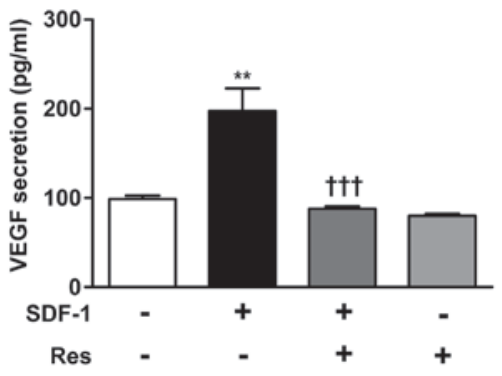

Figure 4. Res suppressed SDF-1-induced VEGF secretion in ARPE-19 cells Cells were pre-treated with Res $(50 \mu \mathrm{M})$ for $2 \mathrm{~h}$ and then co-treated with SDF-1 (30 $\mathrm{ng} / \mathrm{ml})$ and Res $(50 \mu \mathrm{M})$ for $12 \mathrm{~h}$. VEGF secretion in ARPE-19 cells was measured by enzyme-linked immunosorbent assay. Data are represented as the mean \pm standard error of the mean and analyzed using one-way analysis of variance with Dunnett's post-test $(\mathrm{n}=3) .{ }^{* *} \mathrm{P}<0.01$ vs. control and ${ }^{\dagger} \mathrm{P}<0.001$ vs. SDF-1. Res, resveratrol; VEGF, vascular endothelial growth

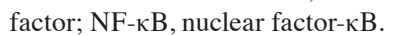

with Normoxia $\mathrm{CM}$ and Res $(50 \mu \mathrm{M})$ served as a negative control (Fig. 5).

\section{Discussion}

In this study, it was determined using $\mathrm{CoCl}_{2}$-induced hypoxia mimetic conditions that there is an association between CXCR4 and VEGF in ARPE-19 cells, and it was assessed whether Res could effectively suppress neovascularization by targeting interactions between CXCR4 and VEGF. Similar to other studies, it was confirmed that CXCR4 and VEGF levels were increased during chemically-induced hypoxia $(24,25)$. Furthermore, it was demonstrated that Res inhibited CXCR4 expression and VEGF secretion, and suppressed HUVEC tube formation.

HIF-1 $\alpha$ is widely used as a hypoxia marker. During hypoxia, HIF- $1 \alpha$ rapidly accumulates as the activation of enzymes responsible for HIF-1 $\alpha$ degradation is reduced (26). $\mathrm{CoCl}_{2}$ is a well-known chemical inhibitor of HIF-1 $\alpha$ degradation (27).
A

a

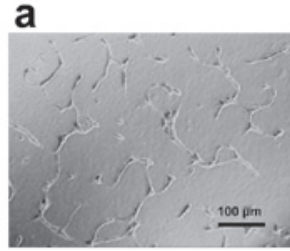

C

Normoxia CM

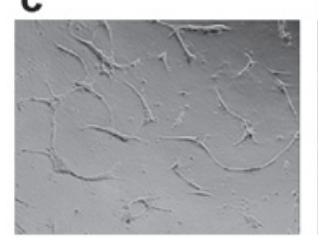

Normoxia $\mathrm{CM}+$ Res

b

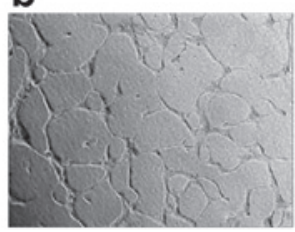

Hypoxia CM

d

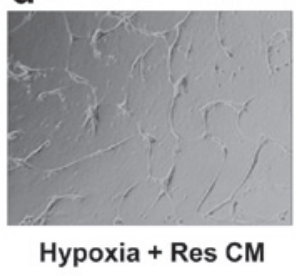

B

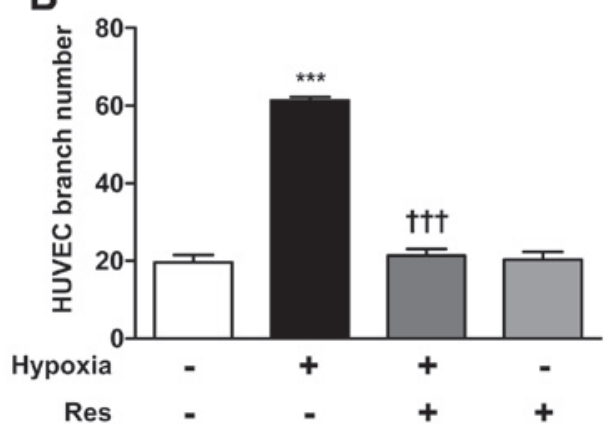

Figure 5. Effects of ARPE-19 cell CM on HUVEC tube formation. (A) Representative images of HUVECs following incubation with ARPE-19 conditioned media. (Aa) Normoxia CM; (Ab) Hypoxia CM $\left(1 \% \mathrm{O}_{2}\right)$; (Ac) Hypoxia+Res CM $\left(1 \% \mathrm{O}_{2}+\right.$ Res $\left.50 \mu \mathrm{M}\right)$; or (Ad) Normoxia $\mathrm{CM}+\operatorname{Res}(50 \mu \mathrm{M})$ for $48 \mathrm{~h}$. (B) Branch numbers in HUVECs were calculated by image software. Data were represented as mean \pm standard error of the mean and analyzed using one-way analysis of variance with Dunnett's post-test $(\mathrm{n}=3){ }^{* * * *} \mathrm{P}<0.001$ vs. Normoxia $\mathrm{CM}$ and ${ }^{\dagger+} \mathrm{P}<0.001$ vs. Hypoxia $\mathrm{CM}$. $\mathrm{CM}$, conditioned media; HUVEC, human umbilical vein endothelial cell; Res, resveratrol.

Therefore, $\mathrm{CoCl}_{2}$-induced HIF-1 $\alpha$ accumulation may result in increased expression of CXCR4 and secretion of VEGF, which is similar to the results obtained following hypoxia.

The $\mathrm{CoCl}_{2}$-induced increase in CXCR4 expression and VEGF secretion was suppressed by Res in a dose-dependent manner. The changes in VEGF secretion paralleled the pattern of CXCR4 expression; therefore, it was hypothesized that CXCR4 is important in the regulation of VEGF secretion. Recent studies verified the interaction between CXCR4 and VEGF in several cell types $(28,29)$. In the present study, Res also suppressed phosphorylation of NF- $\mathrm{kB}$. Certain studies have found that NF- $\mathrm{kB}$ activation is associated with CXCR4 expression $(30,31)$. Furthermore, NF- $\kappa B$ was found to be an important component of the SDF-1/CXCR4 axis (32). NF- $\kappa B$ is a typical transcription factor that is activated through phosphorylation (23). A recent study showed that phosphorylation of NF- $\kappa B$ increases during hypoxia (33). Therefore, suppression of NF- $\mathrm{kB}$ phosphorylation by Res could cause suppression of CXCR4 expression. It was hypothesized that Res inhibits phosphorylation of NF-kB leading to suppression of CXCR4 protein expression, which then affects VEGF secretion. The 
results of PDTC and AMD3100 treatment supported that. $\mathrm{NF}-\kappa \mathrm{B}$ was recently shown to be involved in the regulation of $\mathrm{CXCR} 4$ transcription, suggesting that $\mathrm{NF}-\kappa \mathrm{B}$ regulates CXCR4 at the mRNA level (34).

SDF-1 and CXCR4 are part of a ligand-receptor axis. As SDF-1 is a ligand of CXCR4, an increase in SDF-1 induces CXCR4 activity (35). In the present study, it was found that Res significantly affected the induction of VEGF secretion and mRNA expression (data not shown) by SDF-1. Thus demonstrating that Res affects the ligand-receptor interaction of SDF-1 and CXCR4, through suppression of CXCR4 expression. The present study suggests that Res may be a useful therapeutic agent that suppresses VEGF secretion in RPE cells through CXCR4 inhibition, thus reducing neovascularization.

The present study also provided data supporting the in vitro experiments using the HUVEC tube formation assay to investigate the effects of Res on retinal neovascularization induced by VEGF secreted from RPE cells. VEGF secretion by ARPE-19 cells was triggered by hypoxia and conditioned medium obtained from the treated cells was applied to HUVECs. HUVEC tube formation was significantly increased by the addition of conditioned media from ARPE-19 cells with chemically induced hypoxia suggesting that VEGF secretion from RPE cells substantially affected neovascularization. Moreover, this effect could be inhibited by co-treatment of the ARPE-19 cells with Res. Additional studies have shown that Res effectively inhibits neovascularization in several cancer cell types $(36,37)$.

In conclusion, an increase in CXCR4 expression during $\mathrm{CoCl}_{2}$ chemically induced hypoxia in ARPE-19 cells was confirmed, accompanied by an increase in VEGF secretion. Res suppression of these responses may be through the inhibition of CXCR4 and $\mathrm{NF}-\kappa \mathrm{B}$, suggesting that Res could be used as a therapeutic agent to suppress retinal neovascularization by targeting CXCR4.

\section{Acknowledgements}

This study was supported by a special clinical fund of Gyeongsang National University Hospital in 2008.

\section{References}

1. Neely KA and Gardner TW: Ocular neovascularization: clarifying complex interactions. Am J Pathol 153: 665-670, 1998

2. Chang JH, Garg NK, Lunde E, Han KY, Jain S and Azar DT: Corneal neovascularization: An anti-VEGF therapy review. Surv Opthalmol 57: 415-429, 2012.

3. Chan EC, van Wijngaarden P, Liu GS, Jiang F, Peshavariya H and Dusting GJ: Involvement of Nox2 NADPH oxidase in retinal neovascularization. Invest Ophthalmol Vis Sci 54: 7061-7067, 2013.

4. Engelmann D, Mayoli-Nüssle D, Mayrhofer C, et al: E2F1 promotes angiogenesis through the VEGF-C/VEGFR-3 axis in a feedback loop for cooperative induction of PDGF-B. J Mol Cell Biol 5: 391-403, 2013.

5. Ma JF, Von Kalle M, Plautz Q, -M Xu F, Singh L and Wang L: Relaxin promotes in vitro tumour growth, invasion and angiogenesis of human Saos-2 osteosarcoma cells by AKT/VEGF pathway. Eur Rev Med Pharmacol Sci 17: 1345-1350, 2013.

6. Muller YA, Christinger HW, Keyt BA and de Vos AM: The crystal structure of vascular endothelial growth factor (VEGF) refined to 1.93 A resolution: multiple copy flexibility and receptor binding. Structure 5: 1325-1338, 1997.

7. Ciulla TA, Danis RP, Criswell M and Pratt LM: Changing therapeutic paradigms for exudative age-related macular degeneration: antiangiogenic agents and photodynamic therapy. Expert Opin Investig Drugs 8: 2173-2182, 1999.
8. Cheng SF, Dastjerdi MH, Ferrari G, et al: Short-term topical bevacizumab in the treatment of stable corneal neovascularization. Am J Ophthalmol 154: 940-948, 2012.

9. Campochiaro PA: Retinal and choroidal neovascularization. J Cell Physiol 184: 301-310, 2000.

10. Liu XH, Kirschenbaum A, Yao S, et al: Upregulation of vascular endothelial growth factor by cobalt chloride-simulated hypoxia is mediated by persistent induction of cyclooxygenase- 2 in a metastatic human prostate cancer cell line. Clin Exp Metastasis 17: 687-694, 1999.

11. Wang Y, Huang L, Yang Y, Xu L, Yang J and Wu Y: Effects of autocrine vascular endothelial growth factor (VEGF) in non-small cell lung cancer cell line A549. Mol Biol Rep 40: 3093-3099, 2013.

12. Saint-Geniez M, Kurihara T, Sekiyama E, Maldonado AE and D'Amore PA: An essential role for RPE-derived soluble VEGF in the maintenance of the choriocapillaris. Proc Natl Acad Sci USA 106: 18751-18756, 2009.

13. Wei L, Fraser JL, Lu ZY, Hu X and Yu SP: Transplantation of hypoxia preconditioned bone marrow mesenchymal stem cells enhances angiogenesis and neurogenesis after cerebral ischemia in rats. Neurobiol Dis 46: 635-645, 2012.

14. Salcedo R, Wasserman K, Young HA, et al: Vascular endothelial growth factor and basic fibroblast growth factor induce expression of CXCR4 on human endothelial cells: In vivo neovascularization induced by stromal-derived factor-1alpha. Am J Pathol 154: 1125-1135, 1999.

15. Jin F, Brockmeier U, Otterbach F and Metzen E: New insight into the SDF-1/CXCR4 axis in a breast carcinoma model: hypoxia-induced endothelial SDF-1 and tumor cell CXCR4 are required for tumor cell intravasation. Mol Cancer Res 10: 1021-1031, 2012.

16. Ozcan Cenksoy P, Oktem M, Erdem O, et al: A potential novel treatment strategy: Inhibition of angiogenesis and inflammation by resveratrol for regression of endometriosis in an experimental rat model. Gynecol Endocrinol: Nov 6, 2014 (Epub ahead of print).

17. Li C, Wang L, Huang K and Zheng L: Endoplasmic reticulum strss in retinal vascular degeneration: Protective role of resveratrol. Invest Opthalmol Vis Sci 53: 3241-3249, 2012.

18. Jang M, Cai L, Udeani GO, et al: Cancer chemopreventive activity of resveratrol, a natural product derived from grapes. Science 275: 218-220, 1997.

19. de la Lastra CA and Villegas I: Resveratrol as an antioxidant and pro-oxidant agent: mechanisms and clinical implications. Biochem Soc Trans 35: 1156-1160, 2007.

20. Soufi FG, Mohammad-Nejad D and Ahmadieh H: Resveratrol improves diabetic retinopathy possibly through oxidative stress-nuclear factor $\kappa \mathrm{B}$-apoptosis pathway. Pharmacol Rep 64: 1505-1514, 2012.

21. Huang W, Li G, Qiu J, Gonzalez P and Challa P: Protective effects of resveratrol in experimental retinal detachment. PLoS One 8: e75735, 2013

22. Vin AP, Hu H, Zhai Y, et al: Neuroprotective effect of resveratrol prophylaxis on experimental retinal ischemic injury. Exp Eye Res 108: 72-75, 2013.

23. Busch F, Mobasheri A, Shayan P, Lueders C, Stahlmann R and Shakibaei M: Resveratrol modulates interleukin-1 $\beta$-induced phosphatidylinositol 3-kinase and nuclear factor $\kappa \mathrm{B}$ signaling pathways in human tenocytes. J Biol Chem 287: 38050-38063, 2012.

24. Zhang ZX, Wang YS, Shi YY, et al: Hypoxia specific SDF-1 expression by retinal pigment epithelium initiates bone marrow-derived cells to participate in Choroidal neovascularization in a laser-induced mouse model. Curr Eye Res 36: 838-849, 2011.

25. Carbajo-Pescador S, Ordoñez R, Benet M, et al: Inhibtion of VEGF expression through blockade of Hif $1 \alpha$ and STAT3 signalling mediates the anti-angiogenic effect of melatonin in HepG2 liver cancer cells. Br J Cancer 109: 83-91, 2013.

26. Semenza GL: HIF-1 and mechanisms of hypoxia sensing. Curr Opin Cell Biol 13: 167-171, 2001

27. Piret JP, Mottet D, Raes M and Michiels C: $\mathrm{CoCl}_{2} \mathrm{~V}$, a chemical inducer of hypoxia-inducible factor- 1 and hypoxia reduce apoptotic cell death in hepatoma cell line HepG2. Ann N Y Acad Sci 973: 443-447, 2002.

28. Jin F, Hagemann N, Schäfer ST, Brockmeier U, Zechariah A and Hermann DM: SDF-1 restores angiogenesis synergistically with VEGF upon LDL exposure despite CXCR4 internalization and degradation. Cardiovasc Res 100: 481-491, 2013. 
29. Sun X, Charbonneau C, Wei L, Yang W, Chen Q and Terek RM: CXCR4-targeted therapy inhibits VEGF expression and chondrosarcoma angiogenesis and metastasis. Mol Cancer Ther 12: 1163-1170, 2013.

30. Manu KA, Shanmugam MK, Rajendran P, et al: Plumbagin inhibits invasion and migration of breast and gastric cancer cells by downregulating the expression of chemokine receptor CXCR4. Mol Cancer 10: 107, 2011.

31. Manu KA, Shanmugam MK, Ramachandran L, et al: First evidence that $\gamma$-tocotrienol inhibits the growth of human gastric cancer and chemosensitizes it to capecitabine in a xenograft mouse model through the modulation of NF- $\mathrm{KB}$ pathway. Clin Cancer Res 18: 2220-2229, 2012.

32. Shanmugam MK, Manu KA, Ong TH, et al: Inhibition of CXCR4/CXCL12 signaling axis by ursolic acid leads to suppression of metastasis in transgenic adenocarcinoma of mouse prostate model. Int J Cancer 129: 1552-1563, 2011.

33. Wang G, Chen C, Yang R, et al: p55PIK-PI3K stimulates angiogenesis in colorectal cancer cell by activating NF- $\mathrm{KB}$ pathway. Angiogenesis 16: 561-573, 2013.
34. Arora S, Bhardwaj A, Singh S, et al: An undesired effect of chemotherapy: gemcitabine promotes pancreatic cancer cell invasiveness through reactive oxygen species-dependent, nuclear factor $\mathrm{\kappa B}$ - and hypoxia-inducible factor $1 \alpha$-mediated up-regulation of CXCR4. J Biol Chem 288: 21197-21207, 2013.

35. Weekes CD, Song D, Arcaroli J, et al: Stromal cell-derived factor $1 \alpha$ mediates resistance to mTOR-directed therapy in pancreatic cancer. Neoplasia 14: 690-701, 2012.

36. Kunimasa K, Ohta T, Tani H, et al: Resveratrol derivative-rich melinjo (Gnetum gnemon L.) seed extract suppresses multiple angiogenesis-related endothelial cell functions and tumor angiogenesis. Mol Nutr Food Res 55: 1730-1734, 2011

37. Fouad MA, Agha AM, Merzabani MM and Shouman SA: Resveratrol inhibits proliferation, angiogenesis and induces apoptosis in colon cancer cells: calorie restriction is the force to the cytotoxicity. Hum Exp Toxicol 32: 1067-1080, 2013. 